\title{
Influence of different types of contact hypersensitivity on imiquimod-induced psoriasis-like inflammation in mice
}

\author{
SHUANG BAI ${ }^{1}$, ZHENYING ZHANG $^{2}$, SUCHUN HOU $^{2}$ and XIAOMING LIU ${ }^{1,2}$ \\ ${ }^{1}$ Department of Dermatology and Venereology, The First Affiliated Hospital of Dalian Medical University, Dalian, \\ Liaoning 116011; ${ }^{2}$ Department of Dermatology and Venereology, The University of Hong Kong-Shenzhen Hospital, \\ Shenzhen, Guangdong 518053, P.R. China
}

Received July 2, 2015; Accepted May 3, 2016

DOI: $10.3892 / \mathrm{mmr} .2016 .5299$

\begin{abstract}
It is currently believed that psoriasis and allergic contact dermatitis (ACD) are different diseases; however, they share clinical similarities. The involvement of $\mathrm{T}$ helper 17 (Th17) cells in these disorders provides a novel opportunity to investigate the relationship between them. The present study aimed to determine whether the same or overlapping inflammatory pathways are involved in the two diseases, and the influence of different types of ACD on psoriasis. Compound mouse models of Th1 or Th2-type contact hypersensitivity (CHS) combined with imiquimod (IMQ)-induced psoriasis-like inflammation were established, in order to mimic the characteristics of ACD and psoriasis. Histopathology, immunohistochemistry and cytokine detection in blood serum and tissues were used to compare the differences between the mice treated with IMQ alone or IMQ combined with Th1 and Th2-type CHS. As compared with the IMQ-treated mice or IMQ-treated Th1-type CHS mice, the mice with Th2-type CHS treated with IMQ exhibited more serious psoriasis-like inflammation with increased epidermal thickness and infiltrating cells in the derma. High mRNA expression levels of interleukin (IL)-17, IL-22, IL-23, TNF- $\alpha$ and ROR $\gamma \mathrm{t}$ were detected in back skin lesions. Additionally, high levels of IL-17 and IL-22 in blood serum were detected in IMQ-treated mice combined with Th2-type CHS. The mice treated with IMQ alone, and IMQ treatment combined with Th1-type CHS had a
\end{abstract}

Correspondence to: Dr Xiaoming Liu, Department of Dermatology and Venereology, The First Affiliated Hospital of Dalian Medical University, 222 Zhongshan Road, Xigang, Dalian, Liaoning 116011, P.R. China

E-mail: liuxiaomingdalian@163.com

Abbreviations: ACD, allergic contact dermatitis; IMQ, imiquimod; CHS, contact hypersensitivity; PASI, psoriasis area and severity index; DNFB, dinitrofluorobenzene; FITC, fluorescein isothiocyanate; IL, interleukin

Key words: psoriasis, contact hypersensitivity, allergic contact dermatitis, Th17 cells, IL-23/IL-17 axis comparable psoriasis-like inflammatory response in the back skin. In conclusion, these data demonstrate that Th2-type CHS exacerbated the IMQ-treated psoriatic inflammation of mice via the IL-23/IL-17 axis. Th17 cells and associated pathways may link ACD and psoriasis. Therefore, patients with psoriasis should avoid contact with specific sensitizers, such as fragrance and rubber products, which may induce Th2 polarization.

\section{Introduction}

Psoriasis and dermatitis eczema are common dermatological diseases. They are considered to be two different diseases due to their mechanisms, pathological changes and treatments (1-3). However, clinically the normal skin of patients with psoriasis will develop psoriatic lesions subsequent to elicitation, which is known as the Koebner phenomenon (4). Similarly, if irritated, the plaque lesions of patients with psoriasis will develop dermatitis eczematous changes and lose their inherent characteristics of psoriasis. A previous histology study determined that epidermal thickening and intra- and inter-cellular edema may occur, instead of extended furcella, dermal papilla and Munro's microabscess, which are pathologically described as psoriatic dermatitis (5). Therefore, these two diseases may be associated and may follow the same or overlapping inflammatory pathways (1).

Traditionally, psoriasis is considered as a T helper type 1 (Th1)-dominated skin disease, and an imbalance of Th1 and Th2 cells has been demonstrated in psoriasis patients $(6,7)$. With the discovery of Th17 cells, psoriasis was thought to be mediated by Th1 and Th17 (8). In a previous study, a large number of Th17 cells and cytokines were detected in the skin lesions and serum of psoriasis patients $(7,9)$. The interleukin (IL)-23/IL-17 axis is considered to be important for the development of psoriasis $(10,11)$.

Contact hypersensitivity (CHS) is a delayed-type hypersensitivity mediated by $\mathrm{T}$ cells, which consits of sensitization and elicitation phases, similar to the human allergic contact dermatitis (ACD) model (12-15). Exposure to different types of chemical sensitization may selectively activate $\mathrm{Th} 1$ or Th2 cells, and induce different types of immune response. Exposure to dinitrofluorobenzene (DNFB) can result in Th1-type CHS with high levels of IFN- $\gamma$; however, relatively low levels of Th2 cytokines, IL-4, -5, and -10. Exposure to 
fluorescein isothiocyanate (FITC) can induce Th2-type CHS accompanied by infiltration of Th2 cells $(16,17)$. The importance of Th17 in inflammatory diseases is being increasingly recognized, with Th17 cells and cytokines detected in CHS mouse models and patients with ACD (18-20).

There is a long-standing debate on the susceptibility and intensity to different contact sensitizations in patients with psoriasis and the relationship between psoriasis and atopic disease. Previous studies have presented varied, even contradictory results $(1,21,22)$. The discovery of Th17 provides a novel perspective of psoriasis and ACD, which allows for the examination of the link between the two diseases. Both the diseases involve Th17 and its cytokines. Therefore, the present study aimed to determine whether the IL-23/IL-17 axis facilitates interaction between the two diseases. In order to achieve this, the compound mouse model of combination CHS with imiquimod (IMQ)-induced psoriasis-like inflammation was established. This model was used to explore the influence of different types of CHS on psoriasis-like inflammation, and the the importance of the Th17-associated pathway.

\section{Materials and methods}

Experimental animals. A total of 72 female Balb/c mice (6-8 weeks; $17 \pm 1.5 \mathrm{~g}$ ) were purchased from the Guangdong Medical Laboratory Animal Center (Guangdong, China). The animals were housed at $24^{\circ} \mathrm{C}$ with $40-60 \%$ humidity on a $12-\mathrm{h}$ dark/light cycle, and provided with food and water ad libitum. The current study was approved by the Laboratory Animal Care and Use Committee of Dalian Medical University (Dalian, China). All protocols used in the present study were in compliance with the Dalian Medical University Guidelines for the proper care and use of laboratory animals.

Reagents and kits. IMQ (5\%) cream was purchased from Sichuan Med-Shine Pharmaceutical Co., Ltd., (Sichuan, China). Dibutyl phthalate (DBP; $>99 \%$ ) and FITC were purchased from Sigma-Aldrich (St. Louis, MO, USA). DNFB was purchased from Shanghai Jonln Reagent Co., Ltd. (Shanghai, China). The other chemicals were of analytical grade. Mouse enzyme-linked immunosorbent assay (ELISA) kits for IL-17 and IL-23 were purchased from eBioscience, Inc. (San Diego, CA, USA). Mouse ELISA kits for IL-22 and interferon (IFN)- $\gamma$ were purchased from Dakewe Biotech Co., Ltd. (Shenzhen, China).

Establishment of compound mouse models of Th1 type or Th2 type CHS combined with IMQ-induced psoriasis-like inflammation. Establishment of classic murine models of IMQ-induced psoriasis and CHS was based on previous studies $(23,24)$. Mice received daily doses of $62.5 \mathrm{mg} 5 \% \mathrm{IMQ}$ cream on their shaven backs for 15 consecutive days. Th1-type CHS was induced by the application of $25 \mu 10.5 \%$ DNFB on the shaven abdomens in the sensitization phase (day 7 and 8), and $20 \mu 10.25 \%$ DNFB on each ear in the stimulation phase (day 13) (13). Th2-type CHS was induced by the application of $200 \mu 11 \%$ FITC on the shaven abdomens in the sensitization phase (day 7 and 8), and $20 \mu 10.5 \%$ FITC on each ear in the stimulation phase (day 13) (25). The solvent for DNFB was acetone and olive oil (aOO, 4:1 ratio), and for FITC was acetone and DBP (aDBP, 1:1 ratio). In a total of 72 female $\mathrm{Balb} / \mathrm{c}$ mice, 36 mice were randomly selected to establish the compound mouse model of DNFB-induced Th1-type CHS combined with IMQ-induced psoriasis-like inflammation (Th1-type compound mouse model). The other 36 mice were used to establish the compound mouse model of FITC-induced Th2-type CHS combined with IMQ-induced psoriasis-like inflammation (Th2-type compound mouse model). The mice were divided into 6 groups (n=6/group) in order to establish the following compound mouse models: Group I, control; group II, solvent; group III, Th1 or Th2; group IV, IMQ; group V, IMQ + solvent; group VI, IMQ + Th1 or IMQ + Th2. The detailed grouping and protocol are presented in Fig. 1.

Scoring severity of skin inflammation. Based on a previous study (23), the Psoriasis Area and Severity Index (PASI) was used as a reference for the scoring of IMQ-induced psoriasis model. The back skin area of the mice treated with IMQ was excluded from the overall score. Erythema, scaling and thickening were scored separately on a scale from $0-4$ : 0 , none; 1 slight; 2 moderate; 3 marked; and 4 very marked. The cumulative score (scale: $0-12$ ), including erythema, scaling and thickening was used to determine the severity of the inflammation.

Ear swelling and ear weight calculations. Ear swelling was determined using vernier calipers. Ear edema was expressed as $(\mathrm{R}+\mathrm{L}) / 2-(\mathrm{R} 0+\mathrm{L} 0) / 2$, where R0 and $\mathrm{L} 0$ stand for the thickness of the right and left ear, respectively, prior to elicitation. $\mathrm{R}$ and $\mathrm{L}$ stand for the thickness at $48 \mathrm{~h}$ after elicitation. The ears were punched with a trephine of $4 \mathrm{~mm}$ diameter, and weighed immediately. Increase in ear thickness and ear weight was used to indicate the extent of inflammation.

Histology. Following the treatment, after $48 \mathrm{~h}$ the mice were anesthetized using pentobarbital sodium $(100 \mathrm{mg} / \mathrm{kg}$, intraperitoneally; Sigma-Aldrich). Blood samples were collected from the retro-orbital plexus and the mice were sacrificed by cervical dislocation, then the ears and back skin were collected and fixed overnight in 10\% formalin at room temperature. The tissues were then embedded in paraffin, cut into $6 \mu \mathrm{m}$ sections, and stained with hematoxylin and eosin. Epidermal thickness (acanthosis) and the number of infiltrating cells were assessed using Photoshop CS4 analysis software (Adobe Systems, Inc., San Jose, CA, USA).

Tissue sample preparation. The ears of the mice were cut and homogenized in $10 \mathrm{ml} / \mathrm{g}$ of PBS $48 \mathrm{~h}$ after the end of the treatment. Following centrifugation at $12,000 \mathrm{xg}$ for $10 \mathrm{~min}$ at $4^{\circ} \mathrm{C}$, the supernatant was collected and IL-17 levels were detected by ELISA.

Enzyme-linked immunosorbent assay. Serum samples were obtained by centrifugation of the collected blood samples (5,000 x g, $\left.15 \mathrm{~min}, 24^{\circ} \mathrm{C}\right)$. Serum levels of IL-17, IL-22, IL-23 and IFN- $\gamma$ were determined using an ELISA kits according to the manufacturer's protocols.

RNA isolation and reverse transcription-quantitative polymerase chain reaction ( $R T-q P C R)$. Total RNA was extracted 
Table I. Primer sequences used for polymerase chain reaction.

\begin{tabular}{lll}
\hline Name & \multicolumn{1}{c}{ Forward (5'-3') } & \multicolumn{1}{c}{ Reverse (5'-3') } \\
\hline IFN- $\gamma$ & GAAAATCCTGCAGAGCCAGATT & TGATGGCCTGATTGTCTTTCAA \\
IL-4 & CCCCAGCTAGTTGTCATCCTG & CAAGTGATTTTTGTCGCATCCG \\
IL-17 & CTCCAGAAGGCCCTCAGACTAC & AGCTTTCCCTCCGCATTGACACAG \\
IL-22 & CAGCTCCTGTCACATCAGCGGT & AGGTCCAGTTCCCCAATCGCCT \\
IL-23 & TCCTCCAGCCAGAGGATCACCC & AGAGTTGCTGCTCCGTGGGC \\
IL-6 & CACAAGTCCGGAGAGGAGAC & CAGAATTGCCATTGCACAAC \\
TNF- $\alpha$ & GAACTGGCAGAAGAGGCACT & AGGGTCTGGGCCATAGAACT \\
ROR $\gamma \mathrm{t}$ & CCGCTGAGAGGGCTTCAC & TGCAGGAGTAGGCCACATTACA \\
AHR & GCCCTTCCCGCAAGATGTTAT & TCAGCAGGGGTGGACTTTAAT \\
T-bet & CACTAAGCAAGGACGGCGAA & CCACCAAGACCACATCCACA \\
$\beta$ - actin & TGGAATCCTGTGGCATCCATGAAAC & TAAAACGCAGCTCAGTAACAGTCCG
\end{tabular}

Group I

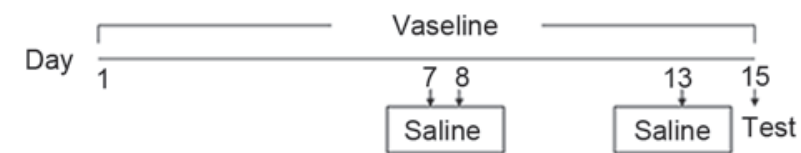

Group II

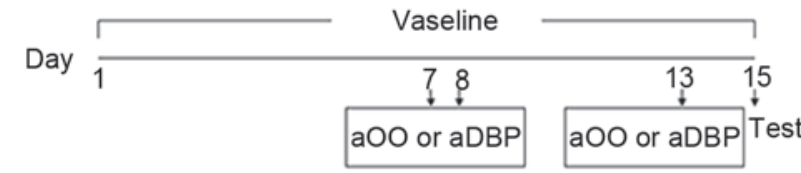

Group III

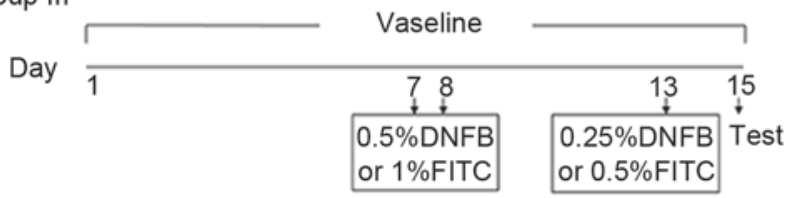

Group IV

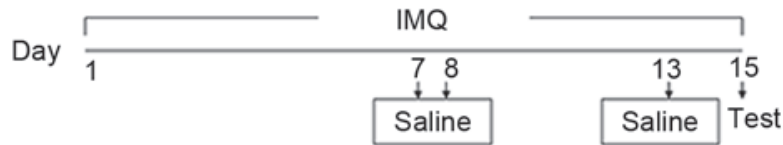

Group V

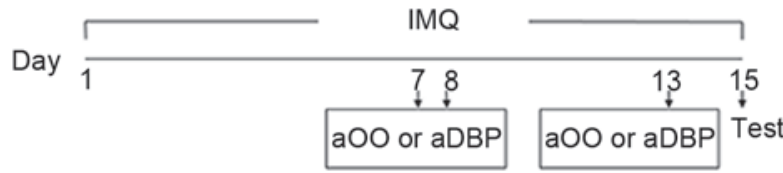

Group VI

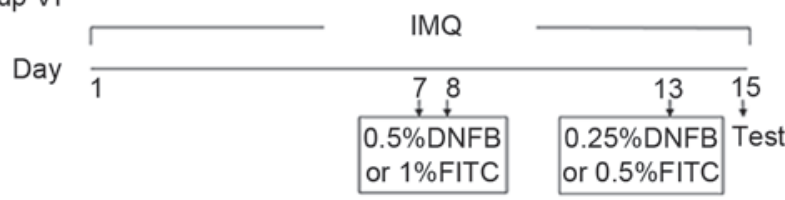

Figure 1. Grouping schedule. Establishment of Th1-type compound murine model or Th2-type compound murine model. Vaseline was applied on the shaven backs of the mice in groups I, II and III for 15 days. IMQ was applied on the shaven backs of mice in groups IV, V and VI for 15 days. The sensitization was performed on days 7 and 8 and elicitation on day 13 by applying saline, solvent, DNFB or FITC solution on the shaven abdomens of mice, depending on treatment groups. Th1, T helper type 1 cells; Th2, Th type 2 cells; IMQ, imiquimod; DNFB, dinitrofluorobenzene; FITC, fluorescein isothiocyanate; aOO, acetone and olive oil; aDBP, acetone and dibutyl phthalate.

from mice ears and dorsal skin biopsies frozen in liquid nitrogen using TRIzol reagent (Invitrogen; Thermo Fisher Scientific, Inc., Waltham, MA, USA). SuperScript III First-Strand Synthesis System (Invitrogen; Thermo Fisher Scientific, Inc.) was used for cDNA synthesis. The reactions were performed on a ViiA7 Real Time PCR System (Thermo Fisher Scientific, Inc.) using FastStart Universal SYBR Green Master (Roche Diagnostics, Basel, Switzerland). The qPCR reaction was conducted as follows: $95^{\circ} \mathrm{C}$ for $10 \mathrm{~min} ; 40$ cycles of $95^{\circ} \mathrm{C}$ for $15 \mathrm{sec}$ and $60^{\circ} \mathrm{C}$ for $1 \mathrm{~min}$. The data were normalized to $\beta$-actin and the relative mRNA levels calculated using the $2^{-\Delta \Delta \mathrm{Cq}}$ method (26). Primer sequences used are indicated in Table I.

Statistical analysis. Statistical analysis was performed with GraphPad Prism 5.01 software (GraphPad Software, Inc., La Jolla, CA, USA). Data are expressed as the mean \pm standard deviation. The differences between experimental groups were analyzed by one-way analysis of variance followed by the
Student-Newman-Keuls post-hoc test. $\mathrm{P}<0.05$ was considered to indicate a statistically significant difference.

\section{Results}

Establishment of the compound mouse model of Th1-type CHS combined with IMQ-induced psoriasis-like inflammation. Ear changes. Following elicitation, signs of ear inflammation such as swelling, capillary expansion and inflammatory cells infiltration were observed in Th1 and IMQ + Th1 groups. Mice in the Th1 and IMQ + Th1 groups had similar degrees of ear inflammation. No obvious changes were observed in the control, solvent, IMQ and IMQ + solvent groups (Fig. 2). The ear swelling and ear weight of Th1 and IMQ+Th1 groups were significantly greater when compared with the control, solvent, IMQ and IMQ+solvent groups (Fig. 2C; P<0.001). There was no significant difference between Th1 and IMQ + Th1 groups (Fig. 2C). 

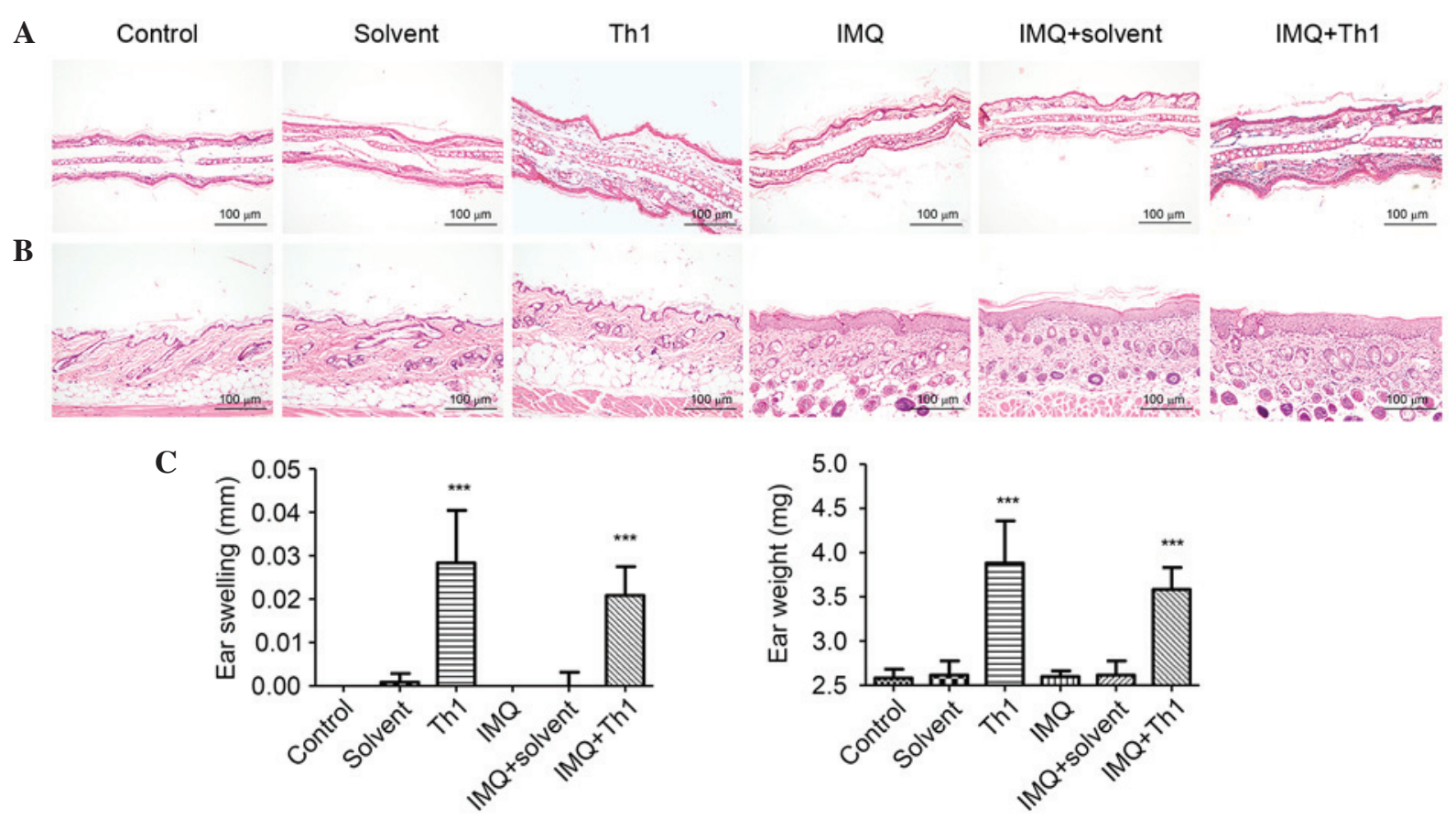

D

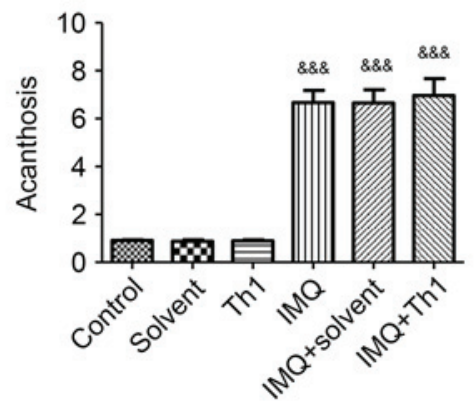

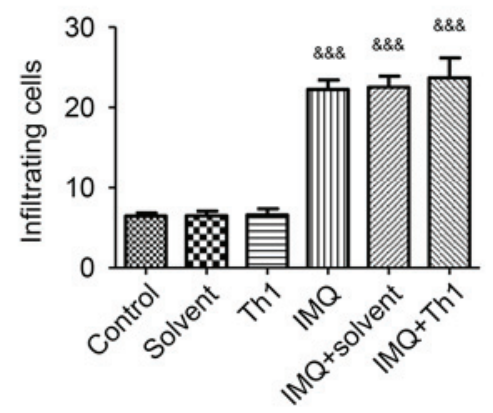

Figure 2. Changes in the ear and back skin in Th1-type compound mouse model. (A) Histological changes in the ear tissues (HE staining). (B) Histological changes in the back skin (HE staining). (C) Ear swelling and ear weight. (D) Acanthosis of the back skin and infiltrating cells in the back skin. ${ }^{* * *} \mathrm{P}<0.001 \mathrm{vs}$. saline or solvent treated groups, ${ }^{\& \&} \mathrm{P}<0.001$ vs. vaseline treated groups. Scale bar $=100 \mu \mathrm{m}$. Th1, T helper type $1 \mathrm{cells}$; HE, hematoxylin and eosin; IMQ, imiquimod.

Back skin changes. Similar to human psoriasis, the back skin lesions of mice in the IMQ, IMQ + solvent and IMQ + Th1 groups presented acanthosis, extended furcella, hyperkeratosis, parakeratosis and infiltration of inflammatory cells. The control, solvent and Th1 groups did not demonstrate any signs of inflammation (Fig. 2B). Acanthosis and the number of infiltrating inflammatory cells in IMQ, IMQ + solvent and IMQ + Th1 groups were significantly increased when compared with the control, solvent and Th1 groups (Fig. 2D; $\mathrm{P}<0.001$ ). There were no significant differences in acanthosis and the number of infiltrating inflammatory cells between IMQ and IMQ + Th1 groups. No significant difference was identified between IMQ, IMQ + solvent and IMQ + Th1 groups (Fig. 2D).

Establishment of the compound mouse model of Th2-type CHS combined with IMQ-induced psoriasis-like inflammation. Ear changes. Mice in Th2 and IMQ + Th2 groups did not exhibit ear inflammatory changes. The ear inflammation in the IMQ+Th2 group was elevated when compared with the Th2 group (Fig. 3). There were no obvious changes in the control, solvent, IMQ and IMQ+solvent groups (Fig. 3A). The mice in the Th2 and IMQ+Th2 groups showed increased ear swelling and ear weight when compared with the control, solvent, IMQ and IMQ+solvent groups (Fig. 3C; $\mathrm{P}<0.001$ ). The mice in the IMQ + Th2 group had enhanced ear swelling and ear weight when compared with the Th2 group (Fig. 3C; $\mathrm{P}<0.05$;).

Back skin changes. The IMQ, IMQ + solvent and IMQ + Th2 groups exhibited psoriasis-like inflammation, whilst the control, solvent and Th2 groups had no obvious changes (Fig. 3B). Acanthosis and the number of infiltrating inflammatory cells in the IMQ, IMQ + solvent and IMQ+Th2 groups were significantly different when compared with the control, solvent and Th1 groups (Fig. 3D; $\mathrm{P}<0.001$ ). The IMQ + Th2 group had increased acanthosis and infiltrating inflammatory cells when compared with the IMQ group (Fig. 3D; $\mathrm{P}<0.05$ ).

Comparison of ear inflammation in Th1 and Th2-type compound mouse models. Histological examination indicated that the ear inflammatory responses in the IMQ + Th1 and IMQ + Th2 groups were comparable (Fig. 4A). There was no significant difference in the ear swelling and ear weight between the two groups (Fig. 4B). The relative mRNA expression level of IFN- $\gamma$ in the IMQ + Th1 group was elevated and significantly higher compared with the IMQ + Th2 group (Fig. 4C; $\mathrm{P}<0.05$ ). However, the difference between the IMQ and IMQ + Th1 
A

Control Solvent

Th2

IMQ

$\mathrm{IMQ}+$ solvent

$\mathrm{IMQ}+\mathrm{Th} 2$
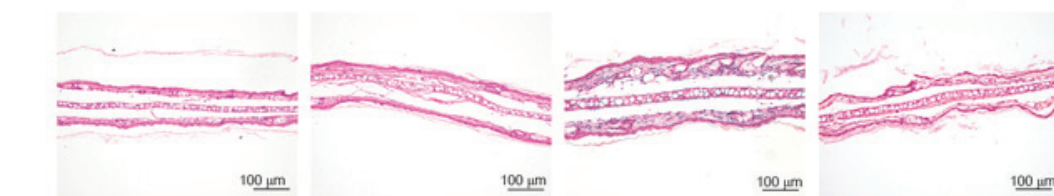

B

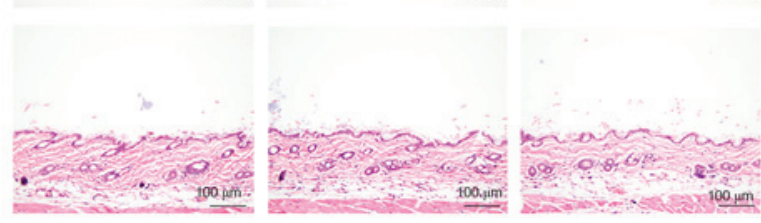

C
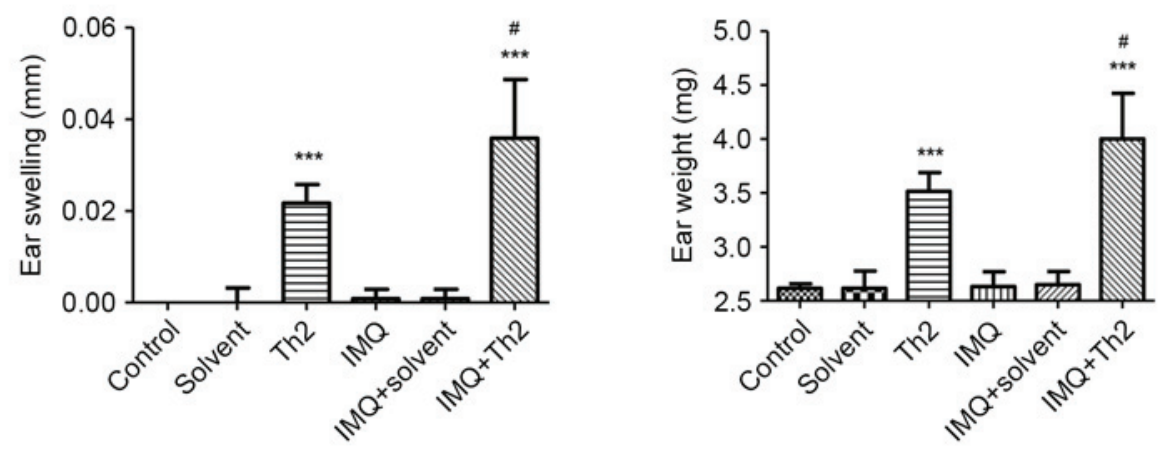

D
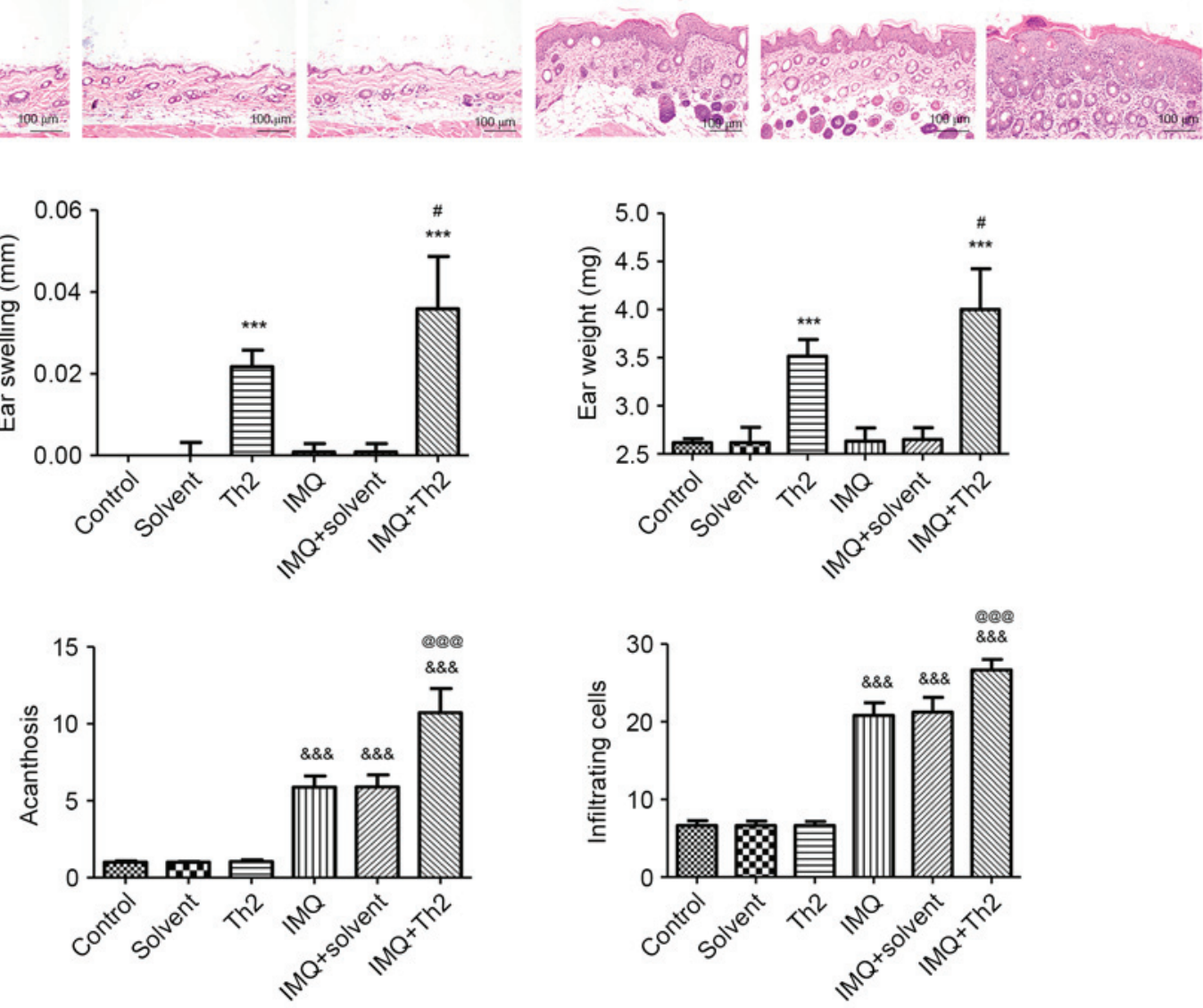
A

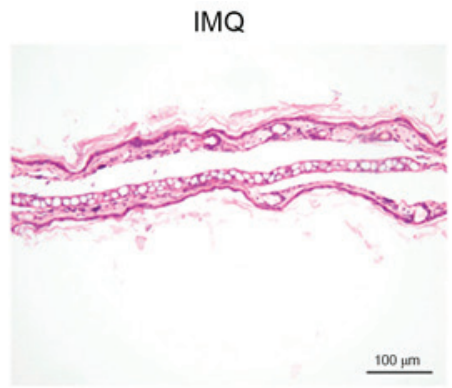

B

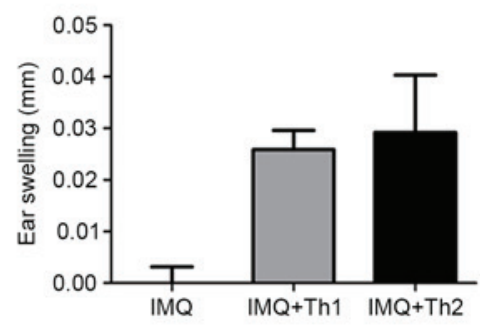

C

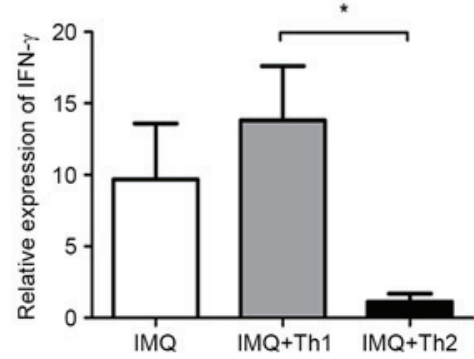

$\mathrm{IMQ}+\mathrm{Th} 1$

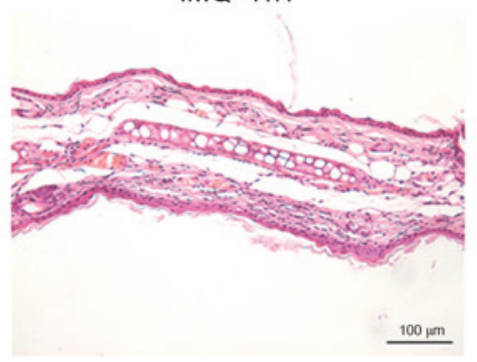

IMQ+Th2
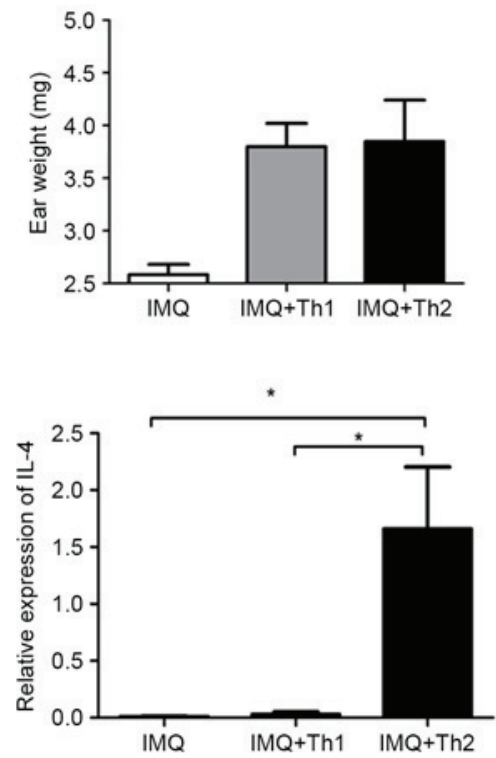

D

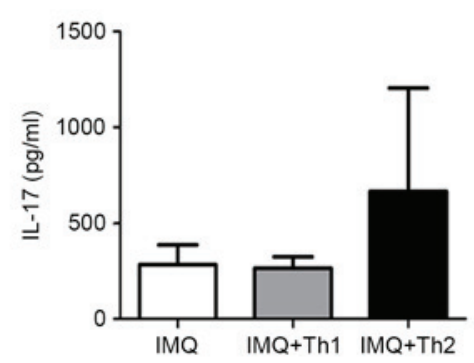

Figure 4. Comparison of ears changes in Th1 and Th2-type compound mouse models. (A) Histological changes in ear tissues (HE staining). (B) Ear swelling and ear weight. (C) Relative mRNA levels of IFN- $\gamma$ and IL-4 in ear tissues. (D) IL-17 level of the ears. Scale bar $=100 \mu \mathrm{m}$. "P<0.05. Th1, T helper type 1; Th2, Th type 2; HE, hematoxylin and eosin; IFN- $\gamma$, interferon- $\gamma ;$ IL, interleukin; IMQ, imiquimod.

groups (Fig. 7A; $\mathrm{P}<0.05$ ), and there were no significant differences between the IMQ and IMQ + Th1 groups (Fig. 7A). Relative mRNA expression levels of IL-23 and TNF- $\alpha$ were significantly higher in the IMQ + Th2 group compared with the IMQ group (Fig. 7A; $\mathrm{P}<0.05$ ). There were no significant differences identified between the IMQ + Th1 and IMQ + Th2 groups (Fig. 7A). There was no significant difference in relative mRNA expression levels of IFN- $\gamma$ and IL- 6 between the IMQ, IMQ + Th1 and IMQ + Th2 groups (Fig. 7A). Relative mRNA expression levels of nuclear hormone receptor retinoic acid-related orphan receptor $\gamma \mathrm{t}(\mathrm{ROR} \gamma \mathrm{t})$, which is a transcription factor specific to Th17 cells, were significantly elevated in the IMQ + Th2 group when compared with the IMQ and IMQ + Th1 groups (Fig. 7B; $\mathrm{P}<0.01 \sim 0.001$ ). The relative mRNA level of ROR $\gamma t$ was significantly higher in the IMQ group when compared with the IMQ + Th1 group (Fig. 7B; $\mathrm{P}<0.05)$. The relative mRNA level of aryl hydrocarbon receptor (AHR), which is the key transcription factor in Th22 cells, was significantly elevated in the IMQ + Th2 group compared with the IMQ + Th1 group. There was no significant difference between the IMQ and IMQ + Th2 groups (Fig. 7B). There was no significant difference in the relative mRNA level of T-bet, which controls IFN- $\gamma$ production and Th1 cell differentiation, between the IMQ, IMQ + Th1 and IMQ + Th2 groups (Fig. 7B).

\section{Discussion}

Based on stable psoriasis-like pathological changes induced by IMQ, mice were sensitized and challenged with DNFB and FITC, which can induce the classic Th1 or Th2-type 
A

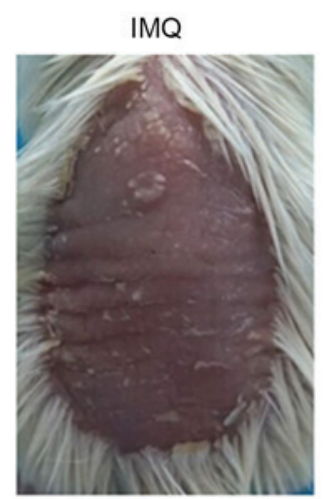

IMQ

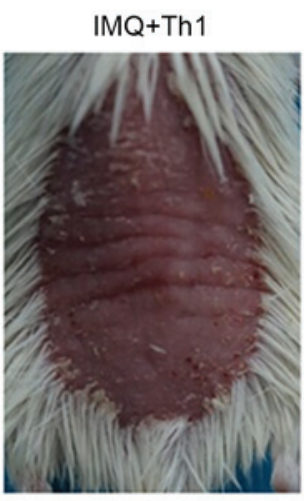

$\mathrm{IMQ}+\mathrm{Th} 1$
$\mathrm{IMQ}+\mathrm{Th} 2$

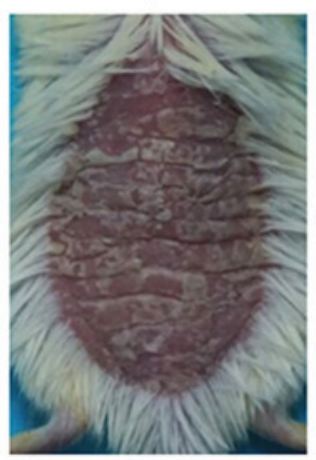

IMQ+Th2

B

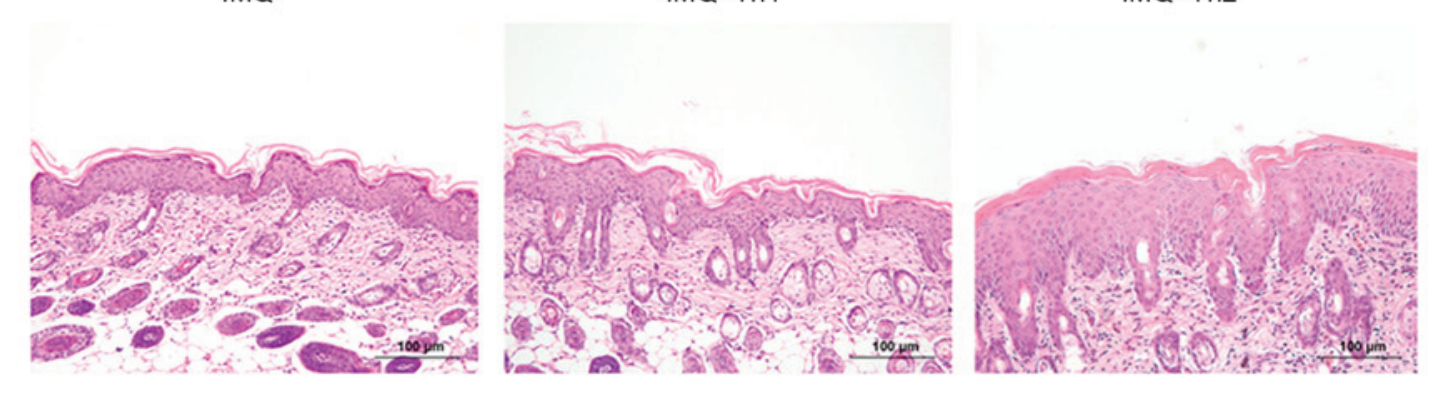

C
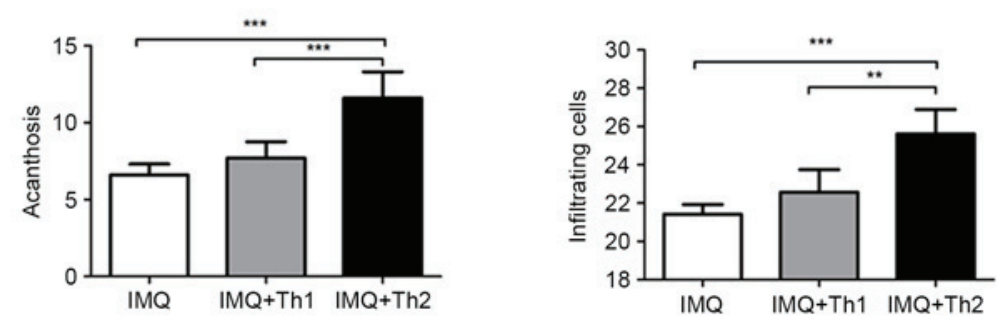

D
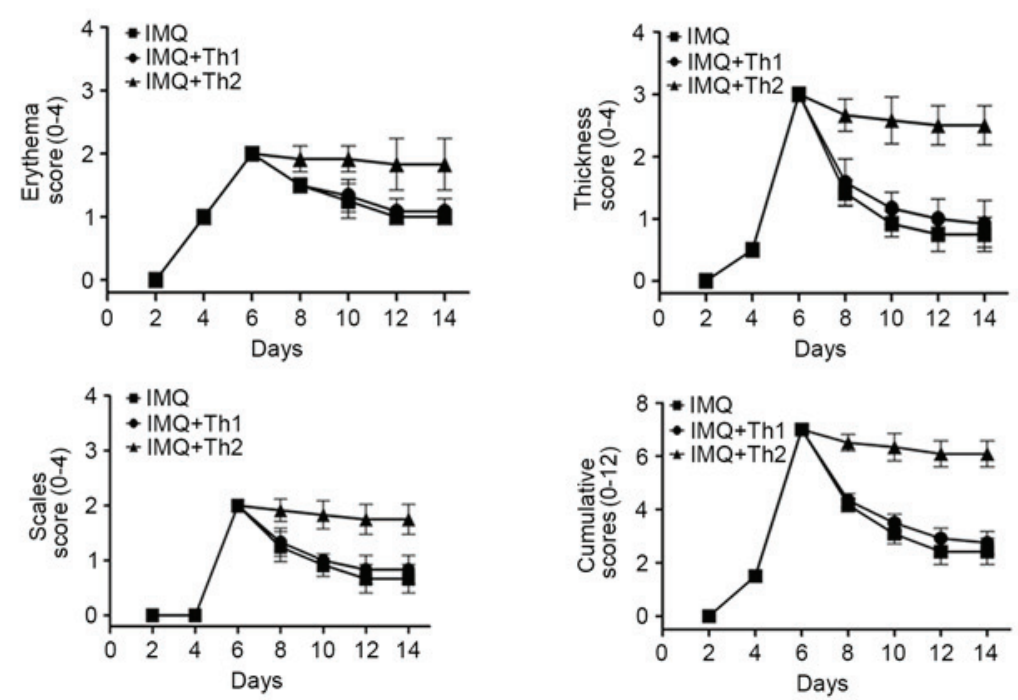

Figure 5. Differences in the skin lesions of Th1 and Th2-type compound mouse models. (A) Representative macroscopic views of the back skin. (B) Histological changes in the back skin (HE staining). (C) Acanthosis and infiltrating inflammatory cells of the back skin. (D) Erythema, scaling and thickness and cumulative scores. Scale bar $=100 \mu \mathrm{m} .{ }^{* *} \mathrm{P}<0.01,{ }^{* * *} \mathrm{P}<0.001$. Th1, T helper type 1; Th2, Th type 2; HE, hematoxylin and eosin; IMQ, imiquimod.

CHS. A total of six subgroups were established to control for the use of saline, vaseline and solvent in the treatments. Through the detection of alterations in morphology, histopathology and cytokines in ear tissues, it was determined that the Th1 or Th2-type CHS mice combined with IMQ-induced psoriasis-like inflammation had a comparable degree of ear inflammation. In the two compound mouse models, CHS was induced by DNFB or FITC and still maintained the inherent 

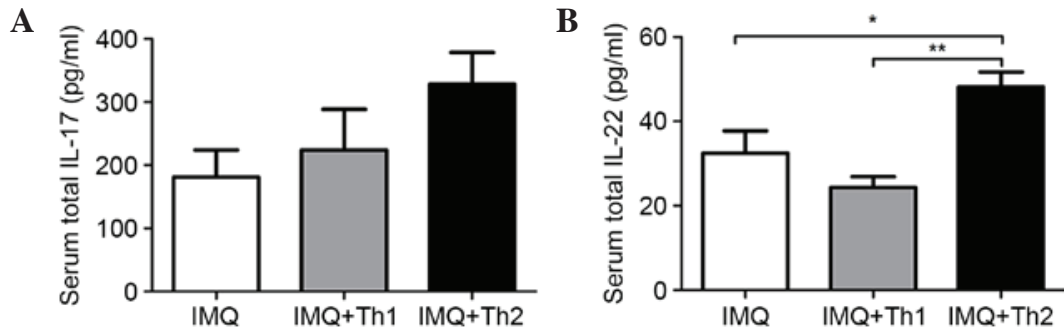

Figure 6. Differences in the serum cytokine levels of Th1 and Th2-type compound mouse models. (A) Serum IL-17 level. (B) Serum IL-22 level. "P<0.05, ${ }^{* *} \mathrm{P}<0.01$. Th1, T helper type 1; Th2, Th type 2; IL, interleukin; IMQ, imiquimod.

A
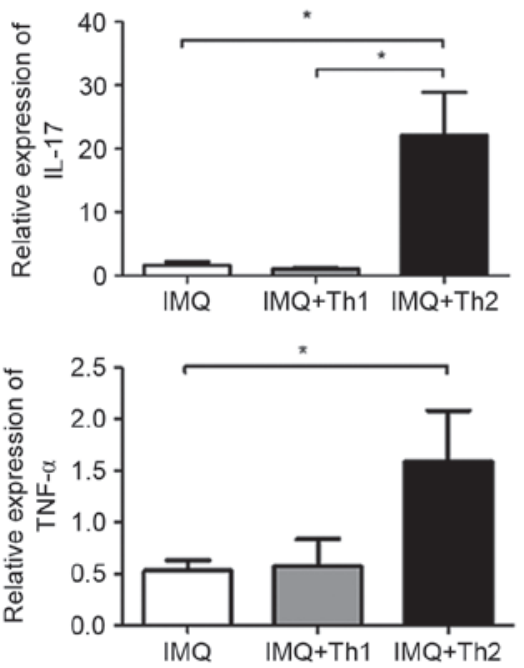

B

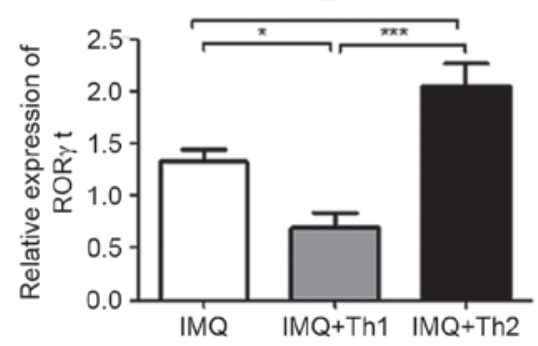

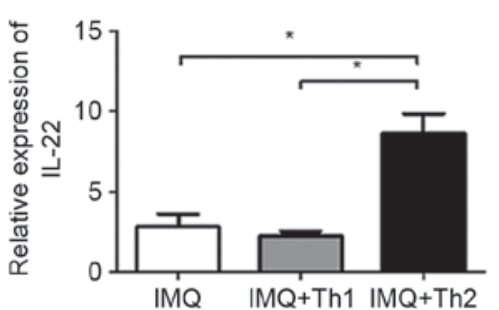
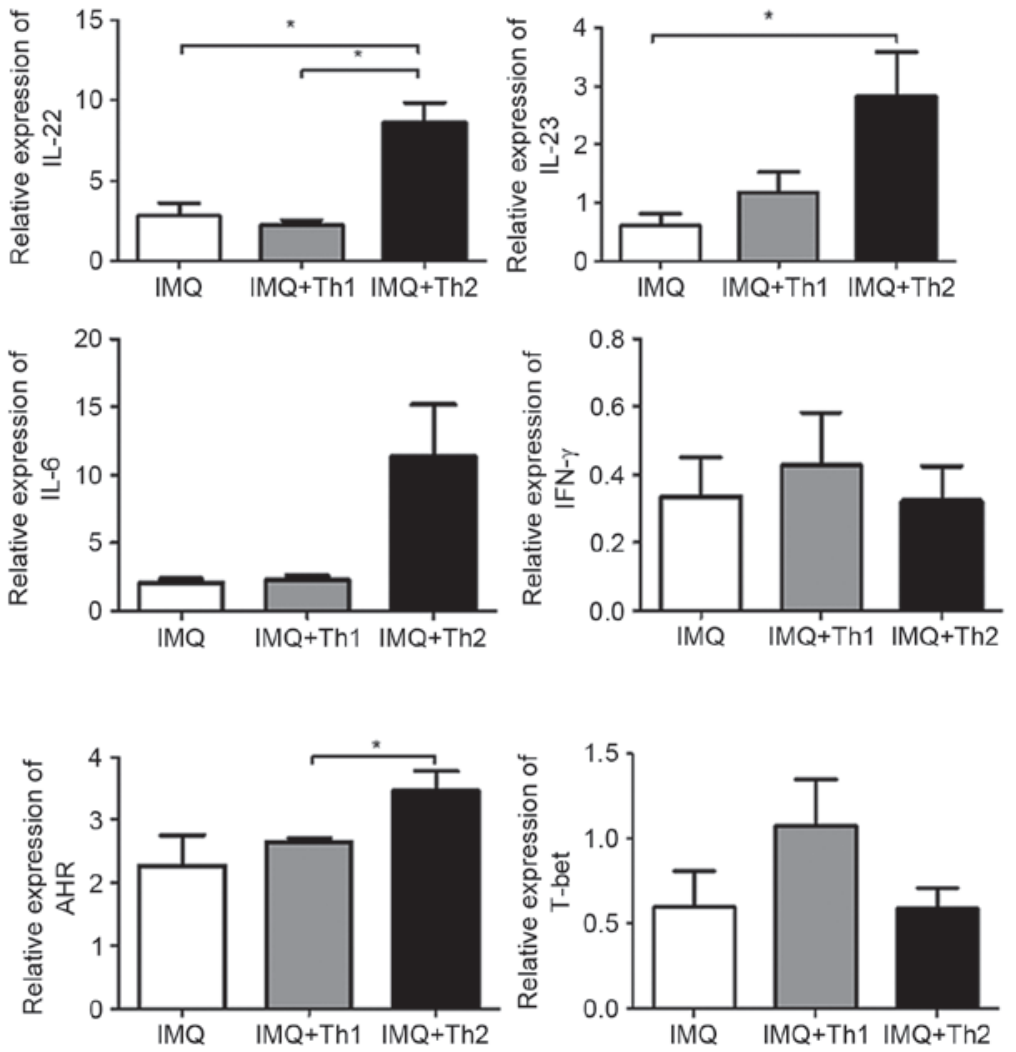

Figure 7. Differences in relative mRNA levels of cytokines and transcription factors in back skin lesions of Th1 and Th2-type compound mouse models. (A) Relative mRNA levels of IL-17, IL-22, IL-23, TNF- $\alpha$, IL-6 and IFN- $\gamma$. (B) Relative mRNA levels of ROR $\gamma \mathrm{t}, \mathrm{AHR}$ and T-bet. ${ }^{*} \mathrm{P}<0.05,{ }^{* *} \mathrm{P}<0.01,{ }^{* * *} \mathrm{P}<0.001$. Th1, T helper type 1; Th2, Th type 2; IL, interleukin; TNF- $\alpha$, tumor necrosis factor- $\alpha$; IFN- $\gamma$, interferon- $\gamma$; ROR $\gamma$ t, nuclear hormone receptor retinoic acid-related orphan receptor $\gamma$ t; AHR, aryl hydrocarbon receptor; T-bet, T-cell-specific T-box transcription factor; IMQ, imiquimod.

inflammatory characteristics. High mRNA expression levels of the Th1 cytokine IFN- $\gamma$ and low mRNA levels of the Th2 cytokine IL-4 were detected in the ears of mice from the combined Th1-type CHS and IMQ-induced psoriasis-like inflammation group. The high mRNA level of IL-4 and low mRNA level of IFN- $\gamma$ were detected in the ears of mice from the combined Th2-type CHS and IMQ-induced psoriasis-like inflammation group. Back skin of the mice also exhibited psoriasis-like inflammation. The histological examination detected epidermal thickening and the infiltration of inflammatory cells in the derma. These results indicate that the clinical and pathological features of psoriasis and allergic contact dermatitis were successfully simulated in the same mouse.

Th17 cells are a novel subset of T helper cells, mainly producing IL-17 and IL-22 (20). Th17 cells are not only responsible for the development of autoimmune diseases but also participate in the pathogenesis of allergic diseases $(19,20,27)$. Previous studies have demonstrated that Th1 cytokines were pathogenic in the development of psoriasis, while Th2 cytokines were protective (28-31). Additionally, studies have indicated that psoriasis is more inclined to be mediated by Th17 than Th1 $(7,32)$. Th17 and the IL-23/IL-17 axis are important for the pro-inflammatory response by coordinating various inflammatory mediators, including IL-6 and TNF- $\alpha(33,34)$. A previous study reported that IL-17A induced Th2 inflammation in acute disease, and $\mathrm{Th} 2$ differentiation from naive $\mathrm{T}$ cells was promoted in vitro by the addition of IL-17A (14). The present study indicated that the mice with combined Th2-type CHS and IMQ-induced psoriasis-like inflammation had marked ear inflammation, histological changes, increased intra- and 
inter-cellular edema, and greater infiltration of inflammatory cells compared with mice with Th2-type CHS. This may be due to Th2-type CHS being exacerbated in the Th17 inflammatory microenvironment presented by IMQ-induced psoriasis-like inflammation.

Previous studies have confirmed that IL-4 is protective in psoriasis, and participates in the negative regulation of Th17 cells $(28,35)$. Therefore, it was hypothesized that Th2-type CHS may relieve psoriasis-like inflammation. By contrast, the results of the present study indicate that Th2 CHS exacerbated the psoriasis-like inflammation induced by IMQ. Although IL-4 participates in the downregulation of Th17, and inhibits the differentiation of Th17 cells, it has also been determined that Th17 cells may develop despite robust Th2 responses (20). The current study determined that the psoriasis-like inflammation was more serious in the mice treated with IMQ combined with Th2-type CHS when compared with mice treated with IMQ + saline. Following sensitization with FITC in the abdomen, the IMQ-treated skin exhibited sustained inflammatory responses and high scores, which demonstrated the deteriorating effect of Th2-type CHS on psoriasis-like inflammation initiated from the sensitization phase. The histological examination indicated that the mice treated with IMQ combined with Th2-type CHS presented thicker epidermis and high infiltration of inflammatory cells compared with mice treated with IMQ + saline, which indicated the persistence and amplification of the psoriasis-like inflammation. High mRNA levels of the Th17 transcription factor, ROR $\gamma \mathrm{t}$, and the associated cytokines, IL-17, IL-22, IL-23 and TNF- $\alpha$, were detected in the psoriasis-like skin lesions. High levels of IL-17 and IL-22 were detected in the serum. However, no obvious changes in mRNA levels of Th1 cell transcription factors, T-bet and IFN- $\gamma$, were observed. This confirmed that the IL-23/IL-17 axis of IMQ-induced psoriatic inflammation was activated by combining with Th2-type CHS, resulting in the exacerbation and amplification of the psoriasis-like inflammation. In a previous study, high levels of thymic stromal lymphopoietin (TSLP), a Th2-polarization cytokine, were detected in the skin lesions of psoriasis patients. TSLP was identified to act synergistically with CD40L and aggravate psoriasis via the promotion of the production of IL-23 (36). Therefore, it is possible that the Th2-associated allergic inflammatory response and psoriasis are not antagonistic. On the contrary, a certain degree of synergistic relationship is observed between Th17 and the IL-23/IL-17 axis; however, the mechanisms remain to be elucidated.

DNFB-induced Th1 CHS has no evident influence on IMQ-treated psoriasis-like inflammation. No differences were observed in the psoriasis-like skin lesions between the mice treated with IMQ + saline and the mice treated with IMQ combined with Th1-type CHS. They had similar skin thickness and comparable levels of infiltration of inflammatory cells. Compared to the the mice treated with IMQ + saline, the mice treated with IMQ combined with Th1-type CHS did not exhibit increased mRNA levels of Th17 transcription factors and associated cytokines in the psoriasis-like lesions or elevated IL-17 and IL-22 levels in serum. Compared with the IMQ + saline group, the IL-23/IL-17 axis was not influenced, indicating that psoriasis is inclined to be mediated by Th17, and Th1 does not served a pivotal role compared with
Th17. There was no alteration to the IFN- $\gamma /$ Th1 response, which may be due to the neutralization of cytokine interactions.

Previous epidemiological studies have reported negative associations between atopy and multiple sclerosis, rheumatoid arthritis and type 1 diabetes $(1,27)$. Autoimmune inflammatory responses tend to suppress the development of atopy, and atopy may suppress the severity of autoimmunity (27). With respect to psoriasis, previous studies have determined that $\mathrm{T}$ lymphocyte function and hapten sensitization were impaired, and the susceptibility to develop contact allergy were decreased in patients with psoriasis $(1,37,38)$. By contrast, previous studies have demonstrated that contact dermatitis was common in patients with psoriasis and there were no statistically significant differences in the contact hypersensitivity and its frequency between plaque psoriasis patients and control groups $(21,22)$. An increased incidence of contact allergy has been observed in patients with palmar-plantar psoriasis and atopy was associated with the protection against the development of psoriatic arthritis $(39,40)$. The results of the current study indicate that different types of CHS differentially influence psoriasis-like inflammation. DNFB-induced Th1-type CHS had no obvious impact on IMQ-treated psoriasis-like inflammation. However, FITC-induced Th2-type CHS exacerbated IMQ-treated psoriasis-like inflammation. Therefore, solely relying on the Th1/Th2 paradigm to explain the relationship between psoriasis and atopic disease is an oversimplification of the disease. It is possible that different sensitizers affect different inflammatory pathways, and differentially influence psoriasis. Dhingra et al (15) identified unique pathways that were preferentially activated by different allergens, which are relevant clinical sensitizers in humans, and determined that exposure to fragrance, and to a lesser extent rubber, resulted in mixed and classic 'allergic' Th2 polarization. Fragrance mix had $100 \%$ current relevance as an aggravating factor of psoriasis, and patients with psoriasis had significant sensitivity to fragrance compared with general dermatology outpatients $(41,42)$. Taken together, these previous studies support the current findings. Therefore, it is recommended that patients with psoriasis should avoid contact with specific sensitizers, such as fragrance and rubber products. We hypothesize that psoriasis and contact dermatitis are not completely independent diseases. They have intrinsic links and Th17-associated pathways may be a point of convergence between the two diseases.

\section{Acknowledgements}

The present study was supported by a grant from the National Natural Science Foundation of China (grant nos. 30872271 and 81171507).

\section{References}

1. Bangsgaard N,Engkilde K, Thyssen JP,Linneberg A, Nielsen NH, Menné T, Skov L and Johansen JD: Inverse relationship between contact allergy and psoriasis: Results from a patient- and a population-based study. Br J Dermatol 161: 1119-1123, 2009.

2. Guttman-Yassky E, Nograles KE and Krueger JG: Contrasting pathogenesis of atopic dermatitis and psoriasis-part I: Clinical and pathologic concepts. J Allergy Clin Immunol 127: 1110-1118, 2011. 
3. Guttman-Yassky E, Nograles KE and Krueger JG: Contrasting pathogenesis of atopic dermatitis and psoriasis-part II: Immune cell subsets and therapeutic concepts. J Allergy Clin Immunol 127: 1420-1432, 2011.

4. Camargo CM, Brotas AM, Ramos-e-Silva M and Carneiro S: Isomorphic phenomenon of Koebner: Facts and controversies. Clin Dermatol 31: 741-749, 2013.

5. Kurz C, Wunderlich S, Spieler D, Schwaiger BJ, Andres C, Traidl-Hoffmann C and Ilg R: Acute transverse myelitis and psoriasiform dermatitis associated with Sjoegren's syndrome: A case report. BMC Res Notes 7: 580, 2014.

6. Jain S, Kaur IR, Das S, Bhattacharya SN and Singh A: T helper 1 to $\mathrm{T}$ helper 2 shift in cytokine expression: An autoregulatory process in superantigen-associated psoriasis progression? J Med Microbiol 58: 180-184, 2009.

7. Martin DA, Towne JE, Kricorian G, Klekotka P, Gudjonsson JE, Krueger JG and Russell CB: The emerging role of IL-17 in the pathogenesis of psoriasis: Preclinical and clinical findings. J Invest Dermatol 133: 17-26, 2013.

8. Lynde CW, Poulin Y, Vender R, Bourcier M and Khalil S: Interleukin 17A: Toward a new understanding of psoriasis pathogenesis. J Am Acad Dermatol 71: 141-150, 2014.

9. Michalak-Stoma A, Bartosińska J, Kowal M, Juszkiewicz-Borowiec M, Gerkowicz A and Chodorowska G: Serum levels of selected Th17 and Th22 cytokines in psoriatic patients. Dis Markers 35: 625-631, 2013.

10. Nakajima K: Critical role of the interleukin-23/T-helper 17 cell axis in the pathogenesis of psoriasis. J Dermatol 39: 219-224, 2012.

11. Works MG, Yin F, Yin CC, Yiu Y, Shew K, Tran TT, Dunlap N, Lam J, Mitchell T, Reader J, et al: Inhibition of TYK2 and JAK1 ameliorates imiquimod-induced psoriasis-like dermatitis by inhibiting IL-22 and the IL-23/IL-17 axis. J Immunol 193 $3278-3287,2014$

12. Honda T, Egawa G, Grabbe S and Kabashima K: Update of immune events in the murine contact hypersensitivity model: Toward the understanding of allergic contact dermatitis. J Invest Dermatol 133: 303-315, 2013.

13. Sugaya M, Kuwano Y, Suga H, Miyagaki T, Ohmatsu H, Kadono T, Okochi H, Blauvelt A, Tamaki K and Sato S: Lymphatic dysfunction impairs antigen-specific immunization, but augments tissue swelling following contact with allergens. J Invest Dermatol 132: 667-676, 2012.

14. Nakajima S, Kitoh A, Egawa G, Natsuaki Y, Nakamizo S Moniaga CS, Otsuka A, Honda T, Hanakawa S, Amano W, et al: IL-17A as an inducer for Th2 immune responses in murine atopic dermatitis models. J Invest Dermatol 134: 2122-2130, 2014.

15. Dhingra N, Shemer A, Correa da Rosa J, Rozenblit M, Fuentes-Duculan J, Gittler JK, Finney R, Czarnowicki T, Zheng X, $\mathrm{Xu} \mathrm{H}$, et al: Molecular profiling of contact dermatitis skin identifies allergen-dependent differences in immune response. J Allergy Clin Immunol 134: 362-372, 2014.

16. Hopkins JE, Naisbitt DJ, Kitteringham NR, Dearman RJ, Kimber I and Park BK: Selective haptenation of cellular or extracellular protein by chemical allergens: Association with cytokine polarization. Chem Res Toxicol 18: 375-381, 2005.

17. Ogawa A, Yoshizaki A, Yanaba K, Ogawa F, Hara T, Muroi E, Takenaka M, Shimizu K, Hasegawa M, Fujimoto M, et al: The differential role of L-selectin and ICAM-1 in Th1-type and Th2-type contact hypersensitivity. J Invest Dermatol 130: $1558-1570,2010$.

18. Larsen JM, Bonefeld CM, Poulsen SS, Geisler C and Skov L: IL-23 and $\mathrm{T}(\mathrm{H}) 17$-mediated inflammation in human allergic contact dermatitis. J Allergy Clin Immunol 123: 486-492, 2009.

19. van Beelen AJ, Teunissen MB, Kapsenberg ML and de Jong EC: Interleukin-17 in inflammatory skin disorders. Curr Opin Allergy Clin Immunol 7: 374-381, 2007.

20. Louten J, Boniface K and de Waal Malefyt R: Development and function of TH17 cells in health and disease. J Allergy Clin Immunol 123: 1004-1011, 2009.

21. Jovanović M, Boza P, Karadaglić D, Brkić S, Petrović A, Mimica-Dukić N, Anackov G and Poljacki M: Contact sensitivity in patients with psoriasis in Vojvodina. Int Arch Allergy Immunol 148: 311-320, 2009.

22. Pigatto PD: Atopy and contact sensitization in psoriasis. Acta Derm Venereol Suppl (Stockh): 19-20, 2000.
23. van der Fits L, Mourits S, Voerman JS, Kant M, Boon L, Laman JD, Cornelissen F, Mus AM, Florencia E, Prens EP and Lubberts E: Imiquimod-induced psoriasis-like skin inflammation in mice is mediated via the IL-23/IL-17 axis. J Immunol 182: 5836-5845, 2009.

24. Qin S, Wen J, Bai XC, Chen TY, Zheng RC, Zhou GB, Ma J, Feng JY, Zhong BL and Li YM: Endogenous n-3 polyunsaturated fatty acids protect against imiquimod-induced psoriasis-like inflammation via the IL-17/IL-23 axis. Mol Med Rep 9: 2097-2104, 2014.

25. Onoue A, Kabashima K, Kobayashi M, Mori T and Tokura Y: Induction of eosinophil- and Th2-attracting epidermal chemokines and cutaneous late-phase reaction in tape-stripped skin. Exp Dermatol 18: 1036-1043, 2009.

26. Livak KJ and Schmittgen TD: Analysis of relative gene expression data using real-time quantitative PCR and the 2(-Delta Delta C(T)) method. Methods 25: 402-408, 2001.

27. Rabin RL and Levinson AI: The nexus between atopic disease and autoimmunity: A review of the epidemiological and mechanistic literature. Clin Exp Immunol 153: 19-30, 2008.

28. Ghoreschi K, Weigert C and Röcken M: Immunopathogenesis and role of T cells in psoriasis. Clin Dermatol 25: 574-580, 2007.

29. Cai Y, Fleming C and Yan J: New insights of T cells in the pathogenesis of psoriasis. Cell Mol Immunol 9: 302-309, 2012.

30. Al-Robaee AA, Al-Zolibani AA, Al-Shobili HA, Kazamel A and Settin A: IL-10 implications in psoriasis. Int J Health Sci (Qassim) 2: 53-58, 2008.

31. Weigert $\mathrm{C}$, Röcken $\mathrm{M}$ and Ghoreschi $\mathrm{K}$ : Interleukin 4 as a potential drug candidate for psoriasis. Expert Opin Drug Discov 3: 357-368, 2008.

32. Nishimoto $S$, Kotani $H$, Tsuruta $S$, Shimizu N, Ito M, Shichita T, Morita R, Takahashi H, Amagai M and Yoshimura A: Th17 cells carrying TCR recognizing epidermal autoantigen induce psoriasis-like skin inflammation. J Immunol 191: 3065-3072, 2013.

33. Singh TP, Schön MP, Wallbrecht K, Michaelis K, Rinner B, Mayer G, Schmidbauer U, Strohmaier H, Wang XJ and Wolf P: 8-methoxypsoralen plus ultraviolet A therapy acts via inhibition of the IL-23/Th17 axis and induction of Foxp3+regulatory T cells involving CTLA4 signaling in a psoriasis-like skin disorder. J Immunol 184: 7257-7267, 2010.

34. Lowes MA, Russell CB, Martin DA, Towne JE and Krueger JG: The IL-23/T17 pathogenic axis in psoriasis is amplified by keratinocyte responses. Trends Immunol 34: 174-181, 2013.

35. Nakagawa R, Yoshida H, Asakawa M, Tamiya T, Inoue $N$, Morita R, Inoue H, Nakao A and Yoshimura A: Pyridone 6, a pan-JAK inhibitor, ameliorates allergic skin inflammation of NC/Nga mice via suppression of Th2 and enhancement of Th17. J Immunol 187: 4611-4620, 2011.

36. Volpe E, Pattarini L, Martinez-Cingolani C, Meller S, Donnadieu MH, Bogiatzi SI, Fernandez MI, Touzot M, Bichet JC, Reyal F, et al: Thymic stromal lymphopoietin links keratinocytes and dendritic cell-derived IL-23 in patients with psoriasis. J Allergy Clin Immunol 134: 373-381, 2014.

37. Moss C, Friedmann PS and Shuster S: Impaired contact hypersensitivity in untreated psoriasis and the effects of photochemotherapy and dithranol/UV-B. Br J Dermatol 105: 503-508, 1981.

38. Bangsgaard N, Engkilde K, Menné T, Løvendorf M, Jacobsen GK, Olsen J and Skov L: Impaired hapten sensitization in patients with autoimmune disease. Clin Exp Immunol 165: 310-317, 2011.

39. Hajdarbegovic E, Nijsten T, Westgeest A, Habraken F, Hollestein L and Thio B: Decreased prevalence of atopic features in patients with psoriatic arthritis, but not in psoriasis vulgaris. $\mathrm{J}$ Am Acad Dermatol 68: 270-277, 2013.

40. Caca-Biljanovska N, V'Lckova-Laskoska M, Balabanova-Stefanova $\mathrm{M}$ and Grivceva-Panovska V: Frequency of delayed-type hypersensitivity to contact allergens in palmo-plantar psoriasis. Prilozi 26: 131-141, 2005.

41. Krupashankar DS and Manivasagam SR: Prevalence and relevance of secondary contact sensitizers in subjects with psoriasis. Indian Dermatol Online J 3: 177-181, 2012.

42. Malhotra V, Kaur I, Saraswat A and Kumar B: Frequency of patch-test positivity in patients with psoriasis: A prospective controlled study. Acta Derm Venereol 82: 432-435, 2002. 